\title{
La privatización de los servicios de inteligencia: ¿Futuro o una vuelta al pasado?
}

\author{
Miguel Anxo Bastos Boubeta \\ UNIVERSIDADE DE SANTIAGO DE COMPOSTELA \\ miguelanxo.bastos@usc.es \\ Alfonso A. López Rodríguez \\ UNIVERSIDADE DE SANTIAGO DE COMPOSTELA \\ alfonsoantonio.lopez@usc.es
}

\begin{abstract}
Resumen: Actualmente se presencian cambios en los paradigmas de inteligencia y seguridad de los Estados, así como la naturaleza cambiante de las amenazas que afrontan en el siglo XXI. Las amenazas emergentes y las necesidades técnicas, tácticas e incluso estratégicas abren un nuevo contexto en el que la acción de entidades privadas en el ámbito de la inteligencia no sólo resulta posible sino deseable. La velocidad del cambio abre la puerta a este tipo de colaboración con la finalidad de obtener una mayor flexibilidad y adaptabilidad estratégica ante los desafíos que encaran los estados. De una forma paradójica este tipo de actividad y relación entre lo público y lo privado en el ámbito de la inteligencia no es una novedad, sino que había existido y se había producido con frecuencia en el pasado.
\end{abstract}

Palabras clave: políticas públicas, inteligencia, seguridad, externalización.

Abstract: Nowadays there are changes in the State intelligence and security paradigms, as well as the changing nature of the threats they face in the XXI century. Emerging threats and technical, tactical needs open a new context in which the action of private entities in the field of intelligence is not only possible but desirable. The pace of change opens the door to this type of collaboration in order to obtain greater flexibility and strategic adaptability to the challenges that States are facing. As a paradox, this type of activity and relationship between public and private entities in the field of intelligence is not a novelty, but it had existed and had occurred frequently in the past

Keywords: public policy, intelligence, security, externalization.

\section{Privatización y servicios de seguridad.}

a prestación de servicios de seguridad en todas sus dimensiones (policía, defensa o inteligencia) parece ser uno de los núcleos irreductibles de la actividad estatal. Incluso teóricos libertarios como Nozick ${ }^{1}$ o Holcombe ${ }^{2}$,partida-

1. Vid., Nozick, Robert (1988) Anarquia, estado y utopía, Fondo de Cultura económica, México.

2. Vid., Holcombe, Randall (2004) «Government: Unnecessary but Inevitable» en Independent Review, vol. 8, no 3, Winter, pp. 325-342. 
rios de la supresión de la intervención estatal en todos sus aspectos, exceptúan a las labores de defensa y protección de la ciudadanía de sus proyectos privatizadores y se definen como minarquistas en vez de anarquistas precisamente porque justifican la propia existencia del estado para la prestación de este tipo de servicios de protección, al entender que no podrían ser prestados de otra forma que a través de un monopolio de carácter público. Las definiciones clásicas del estado, al estilo de la de Max Weber, enfatizan en el monopolio de la violencia y de la protección como su principal característica definitoria. De ahí se comprende que los procesos de privatización de la protección y la defensa que se han producidos en los últimos treinta años ${ }^{3}$ hayan despertado sorpresa, cuando no rechazo, en muchos sectores sociales y originando contestación en ámbitos académicos, en especial en los jurídicos, y politológicos ${ }^{4}$. Nuestra postura, en cambio, apunta a que estas propuestas son fruto de cambios en el entorno , tanto en el ámbito nacional como en el internacional, que condiciona la prestación de dichos servicio, y que estos procesos privatizadores no son más que una adecuación a nuevas realidades y que, en última instancia, no son más que una vuelta, tras un largo paréntesis temporal, a las condiciones en los que estos han sido prestados tradicionalmente a lo largo de la historia sólo que adaptados a las características de nuestra época.

Históricamente la prestación de funciones de seguridad, tanto en lo referido a la defensa del territorio como en el ataque a otros colectivos, han sido prestadas en muchas ocasiones bien por el concurso de tropas a sueldo ya sea mediante la recluta de soldados a cambio de un salario, bien sea mediante la contratación de compañías dedicadas a estos menesteres. Los ejemplos históricos abundan. El historiador Jenofonte en su Anábasis nos relató sus experiencias como mercenario ${ }^{5}$ griego al servicio

3. Vid., Bryden, Alan (2006) «Approaching the Privatisation of security from a Security Governance Perspective» en Alan Bryden y Marina Caparini (eds.), Private Actors and Security Governance, Transaction, New Brunswick, pp. 3-19; Shearer, Daniel (1988) «Outsourcing War» en Foreign Policy, no 112, Fall, pp. 68-81.

4. Véase por ejemplo: Alejandre Sintes, Luis (2009) «Implicaciones de la presencia privada en el planeamiento y ejecución de la misión militar» en Helena Torroja Mateu (dir.) La privatización del uso de la fuerza armada, Bosch Editor, Barcelona, pp. 29-36; Nuñez Villaverde, Jesús A. (2009) «Privatización e internacionalización del uso de la fuerza: los mercenarios como lacra del marco de seguridad del siglo XXI» en Helena Torroja Mateu (dir). La privatización del uso de la fuerza armada, Bosch Editor, Barcelona , pp. 63-82; Markusen, Ann R. (2003) «The Case Against Privatizing National Security» en Governance, vol. 16, no 4, October, pp. 471-501; Minow, Martha (2005) "Outsourcing Power: How Privatizing Military Efforts Challenges Acountability, Professionalism and Democracy» en Boston College Law Review, vol. 46, no 5, pp. 989-1026; Wulf, Herbert (2007)«Privatizing and Internationalizing Violence» en The Economics of Peace and Security Journal, vol. 2, nำ, pp. 35-40; Urbina, Julio Jorge (2008), «EI papel de las compañías militares y de seguridad privadas en los conflictos armados recientes: una aproximación al estatuto jurídico de su personal en el derecho internacional humanitario» en José Julio Fernández Rodríguez, Javier Jordán Enamorado y Daniel Sansó-Rubert Pascual (eds.) Seguridad y defensa hoy: Construyendo el futuro, Plaza y Valdés, Madrid.

5. A lo largo de la exposición del presente trabajo se entenderá (en honor a la simplicidad obligada por el espacio disponible) a todo personal combatiente o no, que realice servicios vinculados con la Defensa de manera 
de los persas. Los ejércitos romanos contaban con abundantes tropas auxiliares compuestas de mercenarios. Mercenarios fueron el Cid Campeador y el Príncipe Negro ${ }^{6}$ en sus correrías por la España medieval, escenario en el que numerosas compañías de guerreros profesionales combatían por encargo, incluyendo a los famosos almogávares conquistadores catalanes de Grecia ${ }^{7}$. Mercenarios y condottieri fueron reclutados por todos los proto-estados del absolutismo, hasta la aparición en tiempos de la revolución francesa de los modernos ejércitos nacionales de recluta. Y mercenarias son buena parte de las tropas que despliegan los Estados Unidos en sus misiones de guerra internacionales ${ }^{8}$ hoy en día $a^{9}$, desde los años 60 en los que comienzan a revi$\operatorname{vir}^{10}$, las que combaten en buena parte, por no decir en todos, de los conflictos bélicos en todos los continentes. Hasta la Guardia Suiza vaticana es un ejemplo viviente de la pervivencia de las tropas mercenarias a lo largo de la historia. Incluso los mares, entre ellos los españoles, fueron protegidos durante siglos por empresarios con patente de corso, y, por cierto, con unos resultados muy superiores a las armadas reales ${ }^{11}$.Más bien cabría decir que en la perspectiva de miles de años de guerras humanas que lo extraño es la existencia de ejércitos de corte nacional, de recluta obligatoria, pues las tropas mercenarias cuentan con numerosas ventajas a la hora de prestar servicios de defensa ${ }^{12}$. No es la menor la de que acostumbran a ser mucho menos sanguinarias que las tropas regulares al combatir con menor pasión y poder llegar a acuerdos más

externa al aparato del Estado. Esto es, como personal no orgánicamente vinculado a la estructura estatal. Esta simplificación, por cuestiones de espacio implica no hacer una distinción entre los contratistas civiles de defensa de aquellos que son mercenarios strictu censo bajo las normas internacionales vigentes. Una aproximación más detallada exigiría una extensión mayor, lo cual no es objeto de análisis del presente trabajo.

6. Sobre las compañías medievales dedicadas al ejercicio de la guerra puede verse de forma novelada: Doyle, Arthur Conan (2010) La compañía blanca, Valdemar, Madrid. Para un relato académico sobre las condiciones de los mercenarios en la época medieval puede consultarse: Contamine, Philippe (1984). La guerra en la Edad Media, Labor, Madrid, 1984.

7. Vid., Setton, Kenneth M. (1985), Los catalanes en Grecia, Orbis, Barcelona

8. Un ejemplo es Blackwater. Vease : Scahill, Jeremy (2010)Blackwater: El auge del ejército mercenario más poderoso del mundo, Planeta, Barcelona.

9. Vid., Smith, Eugene B. (2003) «The New Condottieri and US Policy: The Privatization of Conflict and Its Implications» en Parameters, Winter pp. 104-119; Avant, Deborah D. (2006) «The Privatization of Security: Lessons from Iraq» en Orbis, vol. 50, no 2, Spring, pp. 1-15; Adams, Thomas K. (1999) «The New Mercenaries and the Privatization of Conflict» en Parameters, Summer, pp. 103-116; Stromberg, Joseph (2003)«Mercenaries, Guerrillas, Militias, and the Defense of Minimal States and Free Societies» en Hans-Hermann Hoppe (edit.), The Myth of National Defense, Ludwig von Mises Institute, Auburn, pp. 215-238.

10. Vid., Burmester, H.C. (1978) «The Recruitment and Use of Mercenaries in Armed Conflicts» en American Journal of International Law, vol. 72, no 1, January, pp. 37-56.

11. Vid.,Tabarrok, Alexander (2007) «The Rise, Fall and Rise Again of Privateers» en Independent Review, vol. XI, no 4, Spring, pp. 565-577; Mabee, Bryan (2009), «Pirates, Privateers and the Political Economy of the Violence» en Global Change, Peace \& Security, vol. 21, no 2, June, pp. 139-152.

12. Vid.,Lynch, Tony y Walsh, A.J. (2000) «The Good Mercenary» en Journal of Political Philosophy, vol. 8, no 2, pp. 133-153. Un debate sobre la moralidad de los ejércitos privados puede verse en: Baker, Denae-Peter, (2010) Just Warrior Inc.: The Ethics of Privatized Force, Continuum, London. 
fácilmente, al mismo tiempo que son menos expuestas por sus mandos a situaciones arriesgadas dado que son difícilmente reemplazables y su coste de formación muy elevado, lo que supondría una gran pérdida para la empresa contratista. De hecho, la enorme mortandad en las guerras llevadas a cabo por soldados de recluta (como la primera o la segunda guerra mundial) se debe a que el coste de obtener nuevas tropas es casi nulo para los responsables militares. Además, el mercenario o el corsario tenía un claro incentivo a no causar bajas o destrucciones innecesarias dado que podía obtener rescates por las personas o los bienes capturados. Las batallas de mercenarios parecían más maniobras que batallas reales, pues se saldaban con muy pocas bajas y destrozos. Las tropas mercenarias evitan tener que mantener y formar un ejército permanente y minimizan los riesgos de interferencia política por parte de este, dado que son muy poco propensas a querer tomar el poder una vez victoriosas. Su negocio es otro. La única ventaja de relevancia que podría aducirse a favor de los ejércitos nacionales y en contra de los mercenarios sería de corte moral, derivada de que el soldado de recluta combate por su patria o por un ideal mientras que el mercenario en principio combate por lucro. Aunque esto no es necesariamente así en ninguno de los dos casos. La mayoría de las razones expuestas en su contra ${ }^{13}$ inciden en el peligro de dejar en manos de empresas privadas y por tanto sometidas a criterios de beneficio cuestiones tan delicadas como la seguridad nacional, o en su supuesta falta de respeto a los derechos humanos, todo ello partiendo de una suerte de superioridad moral que vendría de combatir por motivos nacionales. Pero en principio esto dependería de cuales fuesen los objetivos del estado nacional y de si estos son o no morales. En muchas ocasiones las tropas de recluta han sido usadas por sus gobernantes para fines innobles e incluso las ideas que inspiran a la tropa pueden haber sido inmorales también, como cuando las mueven ideales racistas o imperiales, mientras que el mercenario podría, a su vez, estar motivado por razones altruistas o de defensa de una justa causa. No se puede determinar a priori que la moralidad de combatir o realizar labores de inteligencia militar por obediencia a los gobernantes de la nación sea más justa, o mejor, que la de combatir por dinero o aventura.

La seguridad privada no se circunscribe en exclusiva a la defensa externa. También las policías están experimentando un proceso de privatización, muy criticado también ${ }^{14}$, que también parece retrotraerlas a sus orígenes cuando estas estaban organizadas de forma privada o comunal ${ }^{15}$. Antes de la aparición de las policías

13. Vid., Schereier, Fred y Caparini, Marina (2005), Privatising Security: Law, Practice and Governance of Private Military and Security Companies, Geneva Center for the Democratic Control of Armed Forces, Geneva, pp. 82-6.

14. Vid., Zielinski, Mike (1995) «Armed and Dangerous: Private Police in the March» en Covert Action Quarterly, no 54, Fall, pp. 44-50.

15. Vid.,Johnston, Les (1992)The Rebirth of Private Policing, Routledge, London. 
estatales en el siglo XIX en Francia con Fouché y en Inglaterra con Peel ${ }^{16}$, existía todo un complejo entramado de organizaciones dedicadas a la represión del crimen dentro de la comunidad ${ }^{17}$. Organizaciones de persecución del crimen, detectives, vigilantes $^{18}$, organizaciones contra el robo de caballos (que en el siglo XX derivarían en organizaciones encargadas de recuperar coches robados), policías de empresa y milicias ciudadanas encargadas de patrullar las calles mantenían el orden en las sociedades preindustriales e incluso en zonas como el oeste americano que carecían de instituciones estatales dignas de tal nombre ${ }^{19}$. Al igual que en el caso de los ejércitos los estados nacionales modernos procedieron lentamente desde comienzos del siglo XIX a monopolizar los servicios de seguridad ciudadana y, por análogas razones, han implementado en los últimos treinta años un proceso de devolución de la prestación de dichos servicios a entidades de corte privado ${ }^{20}$ quienes poco a poco van asumiendo funciones crecientes en este ámbito ${ }^{21}$ incluida la justicia ${ }^{22}$ y las prisiones $^{23}$, pasando incluso por el alquiler de fuerzas de policía pública uniformada para usos privados ${ }^{24}$.

Si tanto la defensa como la seguridad interna han experimentado desarrollos paralelos no es de extrañar que las funciones de inteligencia hayan seguido también una evolución semejante, dada su estrecha relación con ambas. La recolección de información para usos de seguridad y estrategia es una función tan antigua como el poder político, y como se puede comprobar a lo largo de su historia ${ }^{25}$ tales funciones en la forma de espía o analista ${ }^{26}$ han sido prestadas por expertos que venden su información o su capacidad al mejor postor. La primitiva industria de la inteli-

16. Vid., Kempa, Michael (2016), «The Private Origin of Modern Public Policing» en Rita Abrahamsen y Anna Leander (eds.), Routledge Handbook of Private Security Studies, Routledge, New York, pp. 20-29.

17. Vid.,South, Nigel (1987) «Law, Profit, and Private Persons» en Clifford D. Shearing y Philip C. Stenning (eds.) Private Policing, Sage, London, pp. 72-109.

18. Vid., Little, Craig B. y Sheffield, Christopher P. (1983) «Frontiers and Criminal Justice: English Private Prosecution Societies and American Vigilantism in the Eighteen and Nineteen Centuries» en American Sociological Review, vol. 48, no 6, December, pp. 796-808.

19. Vid., Anderson, Terry y Hill, P.J. (1979) «The Not So Wild Wild West» en Journal of Libertarian Studies, vol. 3, no 1, pp. 9- 29.

20. Vid.,Shapiro, Susan S. (1987)«Policing Trust» en Clifford D. Shearing y Philip C. Stenning (eds.) Private Policing, Sage, London, 1987, pp. 194-220.

21. Véase como ejemplo las policías privadas en numerosas ciudades de Estados Unidos: Dorffi, Christine (1979) "San Francisco's Hired Guns» en Reason, August, pp. 26-33.

22. Vid.,Benson, Bruce (2000) Justicia sin estado, , Union Editorial, Madrid.

23. Vid., Feeley, Malcolm M. (1991)«The Privatization of Punishment in Historical Perspective» en William T. Gormley (ed.) Privatization and Its Alternatives, University of Wisconsin Press, Maison, 1991, pp. 199-225.

24. Vid., Reiss Jr., Albert J. (1991) «Private Employment of Public Police» en William T. Gormley (ed.) Privatization and Its Alternatives, University of Wisconsin Press, Maison, pp. 226-240.

25. Vid., Navarro Bonilla, Diego (2009) iEspías!: tres mil años de información y secreto, Plaza y Valdés, Madrid.

26. Vid.,Goldhammer,H. (1987) The Advisor, Elsevier, New York. 
gencia, desde los primitivos espías o toda la panoplia de chivatos e informadores ${ }^{27}$ que venden sus habilidades a quien esté interesado en pagárselas, ha suministrado la información necesaria a militares y políticos para todo tipo de usos, pero sin constituir agencias especializadas, que no llegarían a florecer hasta comienzos del siglo XX. También han prestado sus servicios a organizaciones privadas interesadas en la información, como los bancos medievales, quienes se cuentan en los primeros en establecer servicios privados de inteligencia ${ }^{28}$. En época tan relativamente próxima como 1861, en el marco de la guerra de secesión norteamericana, el presidente Abraham Lincoln recurrió a los oficios de la agencia de detectives Pinkerton (que además de prestar servicios de policía privada también desempeñaba labores de inteligencia) para espiar y recabar información sobre los movimientos y estrategias de los sudistas $^{29}$. Incluso llegó a ejecutarse como espía a uno de los detectives de Pinkerton, como prueba de que los riesgos que asumen no son distintos de los de un espía estatal. La agencia Pinkerton se configuró de hecho como la primera fuerza de policía que operaba a nivel federal en los Estados Unidos. Otras grandes empresas como Burns, Wackenhut o Intertel desempeñaron y siguen desempeñando hoy funciones integradas en el ámbito de la seguridad y la inteligencia, sobre todo en el mundo corporativo (contraespionaje, protección de ejecutivos de empresas destinados a países conflictivos, inteligencia sindical, formación de cuerpos de seguridad, etc.) $)^{30}$ .A principios del siglo XX comenzaron a institucionalizarse en el mundo occidental agencias de inteligencias de carácter estatal que además de recabar información sobre las estrategias e intenciones de las naciones rivales realizaban todo tipo de acciones encubiertas, incluidas las de subversión ${ }^{31}$. Las grandes agencias estatales triunfan al mismo tiempo que se consolidan los estados nacionales en Europa y América del Norte y consolidan su poder e influencia con las grandes guerras nacionales del siglo $\mathrm{XX}$, la primera y la segunda guerra mundiales. En la era de las rivalidades entre estados y luego de bloques ideológicos es donde estas agencias ahora completamente estatales, conformadas con espías y analistas funcionarios e inspiradas por ideas de carácter patriótico o ideológicas, encuentran un marco adecuado para desarrollar sus

27. Vid.,Beresford, M.W. (1957)«The Common Informer, The Penal Statutes and Economic Regulation» en Economic History Review, vol. 10, no 2, pp. 221-238.

28. Vid., Alem, Jean Pierre (1983), El espionaje y el contraespionaje, Fondo de Cultura Económica, México.

29. Vid.,Horan, James D. (1967) The Pinkertons: The Detective Dynasty That Made History, Bonanza Books, New York, pp. 51-148.

30. Vid., O'Toole, George(1978) The Private Sector: Private Spies, Rent-a-Cops, and the Police-Industrial Complex, W.W. Norton, New York, pp. 20-40; Pozo Serrano, Pilar (2008), « La privatización de la formación militar y policial en los programas internacionales de reforma del sector de la seguridad» en José Julio Fernández Rodríguez, Javier Jordán Enamorado y Daniel Sansó-Rubert Pascual (eds.) Seguridad y defensa hoy: Construyendo el futuro, Plaza y Valdés, Madrid, pp. 177-206.

31. Vid.,Codevilla, Angelo (1992) Informing Statecraft, The Free Press, New York,pp. 355-386. 
funciones y son diseñadas como una agencia administrativa más en sus respectivas administraciones públicas., con su presupuesto y sus directores nombrados con criterios políticos. La seguridad, sobre todo la externa, se define frente a las intenciones consideradas agresivas de otras potencias sea en el ámbito bélico, en el ideológico o en el económico, y es principalmente estatocéntrica, matizada levemente por lealtad a la causa comunista o democrático liberal de los agentes en el caso de la guerra fría ${ }^{32}$. No es de extrañar que las agencias de inteligencias se definan también con respecto a esta definición del enemigo y se diseñen por tanto para combatir a otros agentes estatales. Las ideas de interés nacional, entendido como el refuerzo de la influencia política o económica de nuestro país, se configuran como las inspiradoras del trabajo de los agentes de los servicios de inteligencia, quienes a su vez tienen que lidiar con agentes con motivaciones muy semejantes a las suyas propias. Pero como veremos en los siguientes epígrafes al igual que la defensa tiene que aceptar cambios derivados de la transformación de los conflictos bélicos, los servicios de inteligencia estatales tienen que adaptarse a las nuevas realidades de seguridad del siglo XXI y de ahí que al igual que soldados y policías tienen que adaptarse a contextos no estatales de seguridad, que les están llevando a recuperar las antiguas formas de titularidad privada en el desempeño de sus funciones. En el resto del trabajo analizaremos la adaptación de los servicios de inteligencia a los cambios que en el estado y en la recolección y análisis de inteligencia se han experimentado desde finales del siglo XX hasta hoy, y que consisten primero en la transformación de la guerra, segundo en los cambios en el funcionamiento de las administraciones públicas occidentales, en especial con la privatización de funciones públicas y tercero con la revolución tecnológica en el campo de la recogida y tratamiento de la información necesaria para la inteligencia. Las tres conducen a los servicios de inteligencia a nuevas formas, que no descansan necesariamente en el estado, y que no son más que una actualización de las viejas formas de suministrar información, ya presentes en el mundo preestatal.

\section{La transformación de la guerra y la transformación de la inteligencia}

Las necesidades de seguridad y por supuesto las de inteligencia contemporáneas están pensadas de acuerdo con las definiciones de seguridad que los propios estados establecen. Normalmente están concebidas en un sistema en el que existe competencia, rivalidad, hostilidad y cooperación entre estados y las estrategias de defensa están

32. De hecho, siguen respondiendo a los intereses del estado que las financia. La prueba son los numerosos casos de espionaje entre servicios de inteligencia de países pertenecientes al mismo bloque. Práctica que, por cierto, sigue tan viva como siempre como demuestran las filtraciones de espionaje entre los Estados Unidos y por ejemplo Alemania. 
concebidas para contrarrestar los desafíos que otros estados nos pueden plantear. Pero el mundo de los estados está cambiando rápidamente ${ }^{33}$ con la emergencia de nuevas formas políticas, como las grandes uniones regionales tipo Unión Europea ${ }^{34}$, con la generalización de formas de organización económica como la empresa transnacional y con la difusión de nuevas formas de comunicación como internet en especial en su versiones 2.0 que trascienden las viejas fronteras del estado nación y que son imposibles de abordar por un estado en solitario sin correr el riesgo de quedar aislado económica y culturalmente del resto del mundo. Las amenazas a la seguridad también evolucionan ya no proceden en exclusiva de fuentes estatales ${ }^{35}$.

El historiador militar israelí Martin van Creveld ha dedicado buena parte de su $\mathrm{obra}^{36}$ a estudiar la emergencia de nuevas amenazas a la seguridad de las modernas sociedades y como estas ya no proceden principalmente de actores estatales hostiles, con la excepción de algún estado gamberro que alborota más que causa daños reales, sino de organizaciones con base no estatal que bien desafían a los estados existentes, que ven de esta forma perder su monopolio territorial de la violencia, o bien, y de aquí la novedad, desafían a otras organizaciones similares a ellas. Mafias transnacionales, guerrillas africanas que buscan controlar minas o territorios estratégicos, ciberguerras entre empresas, maras juveniles provenientes de antiguas guerrillas que controlan barrios enteros y sus negocios en Centroamérica ${ }^{37}$, terroristas islámicos organizados en red o recientemente en ejércitos de base transnacional reclutados en internet, modernos piratas con conexiones en la City londinense u organizaciones chantajistas que amenazan con virus, armas químicas, epidemias o catástrofes ecológicas son algunos ejemplos de desafíos a la seguridad de nuestras sociedades ${ }^{38} \mathrm{a}$ las cuales el estado nación con sus ejércitos y armamentos convencionales y sus servicios de inteligencia pensados en clave geoestratégicas o en clave de interés nacio-

33. Vid., Ohmae, Kenichi (1995) The End of the National State; The Rise of Regional Economics, The Free Press, New York.

34. Sobre las repercusiones en el mundo de la inteligencia de la integración europea vease: Pulido Gragera, Julia (2010) «Inteligencia: la savia de la Unión Europea» en Inteligencia y Seguridad, no 7, Diciembre-Mayo, pp. 87-107; Monsalve, Rafael (2012)«¿Hacia una inteligencia Europea?» en José Julio Fernández Rodriguez , Daniel Sansó-Rubert Pascual, Julia Pulido Gragea y Rafael Monsalve (eds.) Cuestiones de inteligencia en la sociedad contemporánea, Ministerio de Defensa, Madrid, pp. 121-126.

35. Vid.,Herman, Michael (2005) «Intelligence and the Revolution in Military Affairs» en Michael Herman, InteIligence Services in the Information Age, Frank Cass, London, pp. 49-64.

36. Véase en especial:Van Creveld, Martin (1991) The Transformation of War, The Free Press, New York.

37. Sobre problemas de seguridad e inteligencia en sociedades post-conflicto vease: Trujillo Álvarez,Pedro (2009) «La falta de institucionalización de la inteligencia y su impacto en la seguridad: el caso de Guatemala» en Inteligencia y Seguridad, no 5, diciembre-mayo, pp. 123-154.

38. Vid.,Lind, William S., Schmitt, John F.,Sutton Joseph W. y Wilson, Gary I. (1989)«The Changing Face of War» en Marine Corps Gazette, October, pp. 22-26. 
nal cerrado no está preparado para combatir adecuadamente ${ }^{39}$. De hecho, según el propio van Creveld, dado que el estado moderno surge como respuesta a los condicionantes de las guerras entre estados nacionales no es de descartar que la propia existencia del estado nación pueda llegar a ser cuestionada a medio plazo y ser sustituida por nuevas formas políticas más adecuadas a las nuevas amenazas ${ }^{40}$. También la forma de combate ha cambiado sustancialmente. De usar formas de guerra «duras» con aviones, bombas o tanques se ha pasado a formas de combate nuevas como las ciberguerras a través de medios informáticos ${ }^{41} \mathrm{o}$ los ataques financieros que pueden destruir las economías de países hostiles sin disparar una sola bala, como algunos apuntan que estaría ocurriendo con los ataques al rublo ruso por ejemplo ${ }^{42}$. Estas nuevas formas de guerra financiera requieren de expertos en finanzas capaces de usar técnicas como la especulación a la baja o de usar precios engañosos, capaces como diría James Rickards ${ }^{43}$, uno de sus principales teóricos, de destruir un país con derivados financieros tipo CDS (credit default swaps). Esto da lugar a la aparición de juegos de guerra financieros y a la aparición de nuevos tipos de inteligencia, encomendado a expertos en finanzas, como el llamado MarkInt,o a expertos en la llamada geoeconomía ${ }^{44}$. El paradigma de conflicto bélico ha pasado, de este modo de las visiones propias del «hard power» a manifestaciones diferentes que adoptan términos como guerra asimétrica o híbrida. Dentro de ellas los movimientos de insurgencia ${ }^{45}$ acaban acaparando el protagonismo que antaño mantenían los ejércitos regulares y, por ende, siendo motivo de atención preferente por las redes de inteligencia (García Guindo, 2013).

De la misma forma que la guerra tiene que adaptarse a los nuevos desafíos con la contratación de empresas privadas de defensa, los servicios de inteligencia tienen que adaptar sus funciones de recolección, tratamiento y análisis de información a las

39. Vid., Lahneman, William J. (2010) «The Need for a New Intelligence Paradigm» en International Jounal of Intelligence and CounterIntelligence, vol. 23, pp. 201-225; Liaropoulos, Andrew N. (2006)A (R)evolution in Intelligence Affairs? In Search of a New Paradigm, Rearch Paper nㅇ 100, Research Institute for European and American Studies, Athens, June.

40. Vid., van Creveld, Martin (1999), The Rise and Decline of the State, Cambridge University Press, Cambridge, pp. 336-421.

41. Vid., Clarke, Richard A. y Knake, Robert K. (2011), Guerra en la red: Los nuevos campos de batalla, Ariel, Barcelona.

42. Vid.,Zarate, Juan C. (2014), «The Coming Financial Wars» en Parameters, vol. 43, no 4, Winter.

43. Vid., Rickards, James (2012), Currency Wars, Penguin, New York.

44. Vid., Olier Arenas, Eduardo (2013) «Inteligencia estratégica y seguridad económica» en Cuadernos de Estrategia, no 162, pp. 9-33.

45. Cabe destacar que los propios movimientos de esta naturaleza son no-estatales, aunque competidores del Estado en -ámbito de los recursos y de las mismas bases de estatalidad como ha señalado el citado García Guindo (2014). Esto los convierte en un factor de detracción de recursos directos e indirectos del Estado. Directamente por su acción de insurgencia, e indirectamente por la corriente de recursos que el Estado a de dedicar a combatirlos, dado que, como indica dicho autor, este tipo de conflictos se convierten en una «guerra larga» (García Guindo, 2014:50). 
nuevas demandas sociales. Por ejemplo si para defenderse de los piratas que asolan las aguas del Índico las grandes marinas de guerra occidentales muestran poca adecuación, dada la asimetría en la forma de funcionar de los actores implicados, y muchos armadores están optando por la contratación de mercenarios que defiendan sobre el terreno los propios buques, lo normal es que los servicios de inteligencia se centren en las relaciones financieras entre los piratas y sus casas matrices situadas en respetables plazas financieras del primer mundo. Para ello es más útil un equipo de contables o de expertos en finanzas que un servicio de inteligencia convencional no pensado para afrontar este tipo de problemas ${ }^{46}$. Tampoco el terrorismo ahora es un terrorismo de origen completamente doméstico, como lo era hasta hace poco y el análisis de inteligencia de organizaciones criminales del tipo Al Qaeda ya no se pueden llevar a cabo con base en un sólo país. Es necesario cooperar en red con otros servicios de inteligencia, que a su vez pueden ser potencialmente hostiles, o bien confiar en organizaciones privadas de corte transnacional y también con estructura reticular que se encarguen de elaborar análisis de inteligencia sobre estos desafíos. Conviene a este respecto recordar que prácticamente todas las compañías de seguridad privada prestan también servicios de inteligencia y cuentan con unidades especializadas en tales menesteres con informaciones en muchos casos sobre el terreno, dado que son también en muchos casos ellas las encargadas de afrontar tales amenazas y dado que buena parte de sus efectivos son antiguos miembros de los servicios de inteligencia estatales. En el caso de la inteligencia empresarial la utilidad de los servicios de inteligencia privados es si cabe aún más evidente. Nadie puede conocer mejor las necesidades de protección de una empresa que otra empresa y sobre todo en el caso de las modernas empresas multinacionales que escapan a la jurisdicción de un único estado, dado que cuentan en muchas ocasiones con divisiones autónomas en países distintos. Además, las amenazas en el ámbito de la inteligencia económica suelen provenir de otras empresas, no de estados, por lo que el diseño de medidas de contrainteligencia será mucho más efectivo si está adecuado a la realidad de la amenaza. Es discutible también si los servicios estatales de inteligencia deberían usar sus recursos en las necesidades específicas de protección de una empresa determinada, por relevante que esta sea.

Las agencias privadas de inteligencia están volviendo al mundo pre-estatal en el que existía una pléyade de soberanías entrecruzadas, con multitud de actores intervi-

\footnotetext{
46. Un ejemplo de esta mala adecuación fue el encargo que el gobierno español realizó al CNI de averiguar si había movimientos desestabilizadores por parte de agentes extranjeros hostiles detrás de los movimientos especulativos de la deuda soberana española en los mercados de deuda. Tras una concienzuda investigación por parte de agentes con escaso conocimiento de finanzas se llegó a la misma negativa conclusión a la que llegaría cualquier inversor experto en tales mercados, de que los movimientos se debían a la falta de confianza en las medidas de ajuste emprendidas por el gobierno español.
} 
nientes sin una jerarquía clara, usando tecnologías del siglo XXI que abaratan y posibilitan la adaptación a estas aún emergentes y poco consolidadas realidades post-estatales. La escala de las amenazas ya no tiene forma ni dimensión de escala estata ${ }^{47}$ por lo que es lógico que las agencias encargadas de confrontarlas adopten escalas también distintas. Por un lado si se dan fenómenos de regionalización económica y política tipo Unión Europea lo normal será que sus servicios de inteligencia se adapten y coordinen y por otra si las amenazas a la seguridad ya no provienen en su mayoría de otros estados sino de entes de carácter privado lo normal será que sean organizaciones de inteligencia de corte privado las actuantes y que estas procedan de las organizaciones que se encargan de realizar buena parte de las operaciones sobre el terreno contra los nuevos enemigos. Otros dos factores de cambio en la forma de librarse las guerras modernas serán de relevancia a la hora de explicar la evolución hacia formas privadas de prestar servicios de inteligencia. La primera son los cambios en la demanda de inteligencia, al cambiar el cliente interesado en tales servicios. Hasta el fin de la guerra fría el principal demandante de inteligencia elaborada era el estado nación que buscaba obtener la información estratégica necesaria para afrontar las amenazas de otros estados potencialmente rivales o hostiles, mientras que a partir de entonces se amplía el espectro de potenciales demandantes de este tipo de información. Empresas transnacionales de tipo comercial o ONG, incluida la Iglesia Católica pionera desde hace mucho tiempo en la obtención de inteligencia para usos no específicamente estatales, comienzan a demandar información relevante para el desempeño de sus negocios o misiones de paz (las ONG son también uno de los principales contratistas de fuerzas «mercenarias»), que incluyen desde la estabilidad económica de un país a informaciones relevantes sobre los conflictos internos dentro de las camarillas dominantes, posibilidad de conflictos étnicos o nacionalistas, y sobre todo inteligencia y contrainteligencia específicas a respecto de empresas u organizaciones potencialmente competidoras. Por razones de ética democrática, pues no parece muy edificante usar recursos públicos para investigar empresas o países competidores para defender intereses privados, o por razones de eficacia, dado que la especialización principal de los servicios de inteligencia convencionales es la defensa y no el análisis económico-político estos últimos parecen estar adaptándose cada vez más al papel residual de analizar las cada vez más escasas amenazas bélicas de origen estatal o terrorista, dejando la inteligencia económica en manos de empresas de inteligencia privadas.

El segundo punto a destacar es que las nuevas amenazas a la seguridad están cada vez menos motivadas, en primer lugar, por intereses geoestratégicos o estatales

47. Vid.,Abrahamsen, Rita y Williams, Michael C. (2009)«Security Beyond the State: Global Security Assemblages in International Politics» en International Political Sociology, vol. 3, pp. 1-17. 
y cada vez más motivadas por intereses económicos de corte privado, como mafias, piratería de buques comerciales y espionaje industrial y cuya dimensión es de escala empresarial o regional y no estrictamente estatal y, en segundo lugar están cada vez más motivadas por intereses de corte ideológico-religioso como organizaciones terroristas que operan en espacios dimensión no estatal como puede ser el mundo islámico en su totalidad. Estos nuevos enemigos no buscan tanto derrotar a los estados en cuanto estados sino a sus organizaciones competidoras, que en pocas ocasiones son los propios estados, tanto como expandir sus credos, por lo que los servicios de inteligencia tradicionales no están ni dimensionados, pues su base de actuación es estatal, ni motivados para luchar contra enemigos que no son exactamente los suyos. Lo dicho hasta ahora no implica que la experiencia de los servicios tradicionales pueda ser desechada, de hecho, buena parte de su experiencia y capacitación es usada por las nuevas agencias privadas, que reclutan numerosos agentes de inteligencia del sector público, solamente que los servicios de inteligencia de base estatal necesitarían ser reconvertidos o externalizados, para adecuarse a las nuevas necesidades de combate ideológico y económico propios de los nuevos desafíos a la seguridad.

\section{Tendencia privatizadora en las administraciones públicas y los servicios admi- nistrativos de inteligencia}

Desde finales de los años 70 se han impuesto, a lo largo y ancho del mundo, numerosos programas de privatización de empresas y servicios públicos. Este proceso comenzó a principios de los años 80 en los Estados Unidos, con la creación de la Grace Commission encargada de revisar los costes del gobierno norteamericano e indicar que actividades podrían hacerse descansar en el sector privado y al poco tiempo fue seguida por numerosos programas en el mismo estilo en la administración conservadora británica de Margaret Thatcher. Estos programas se extendieron rápidamente a otros países y fueron incluso asumidos por partidos socialistas, hasta el punto de que los programas más ambiciosos de privatización se llevaron a cabo bajo una administración laborista en Nueva Zelanda.

Los servicios de inteligencia son y están organizados como una administración pública más en todo el mundo ${ }^{48}$. Lo que difiere de otras es la peculiaridad de sus cometidos y una mayor opacidad en su ejecución presupuestaria, pero cuentan con una dirección nombrada políticamente, un personal de plantilla organizado jerárquicamente y reciben sus ingresos de los presupuestos como cualquier otra administración. No extraña pues que desde los comienzos del proceso hayan sido afectadas

48. Vid., Ruiz Miguel, Carlos (2002)Servicios de inteligencia y y seguridad del estado constitucional, Tecnos, Madrid, 2002; 
por este y se hayan privatizado o externalizado buena parte de las funciones antes prestadas en exclusiva por organizaciones públicas ${ }^{49}$. Antes de analizar las formas en que esta se ha desarrollado conviene distinguir la privatización de servicios del uso para fines particulares de los servicios de inteligencia, tal como algún autor ha denunciado ${ }^{50}$. En este último caso no se trata de una verdadera privatización sino de un uso espurio de los servicios públicos de inteligencia en beneficio privado de directivos corruptos o de empresas a estos asociados. De los tres grandes tipos de privatización $^{51}$, que se pueden distinguir en la teoría, la venta total o parcial de activos, la prestación externa de servicios y la retirada del estado de la prestación (que sería en sentido estricto la verdadera privatización dado que los servicios externalizados siguen siendo financiados con fondos públicos y atienden en principio al interés definido en cada momento como general).La privatización de los servicios de inteligencia se ha centrado en los dos últimos dado que no se han registrado ventas de activos o de unidades de inteligencia. En efecto la llamada privatización de la inteligencia se ha producido básicamente en el ámbito de la contratación externa de servicios de inteligencia a empresas establecidas para tal fin que configuran el ahora conocido como Complejo Inteligencia-Industrial ${ }^{52}$, compuesto de empresas privadas y organizaciones públicas entrelazadas ${ }^{53}$, y cuyo principal mercado es el norteamericano pues es el primer consumidor de inteligencia del mundo, y se ha extendido a otros territorios, en especial al resto de países occidentales. En países como los Estados Unidos la mayor parte de los fondos públicos dedicados a inteligencia, entre el 60 y el 70\%, son destinados a empresas integradas en este complejo. Este incluye desde grandes empresas que prestan servicios integrados, como las líderes en el sector Booz Allen Hamilton y SAIC, con varios miles de empleados cada una de ellas, y las divisiones de inteligencias de empresas como Raytheon, Lockheed Martin o Computer Sciences Corporation. Son estas las contratistas de los mega-proyectos del gobierno. Estas grandes empresas de inteligencias subcontratan determinados servicios muy especializados a empresas medianas del sector, tipo CACI International o ManTech Intenational como programas de software o especialistas en telecomunicaciones. Además, existen en el último escalón un abundante conjunto de pequeñas empre-

49. Vid., Rathmell, Andrew (1998) «Privatising Intelligence» en Cambridge Review of International Affairs, vol. $\mathrm{XI}, \mathrm{n}$ 을 , Spring, pp. 199-211.

50. Vid., Trento, Joseph T. (2005) Prelude to Terror: The Rogue CIA and the Legacy of America's Private Intelligence Network, Carroll and Graf Publishers, New York.

51. Vid, Savas, E.S. (2001) Privatization and Public Private Partnerships, East-West Press, New Delhi, pp. 125137; Donahue,John D. (1991) La decisión de privatizar. Fines públicos y medios privados, Paidós, Barcelona.

52. Vid.,Shorrock, Tim (2009)Spies for Hire: The Secret World of Intelligence Outsourcing, Simon \& Schuster, New York, pp. 9-37; Keefe, Patrick, R. (2010), «Privatized Spying: The Emerging Intelligence Industry» en Loch Johnson (edit.), The Oxford Handbook of National Security Intelligence, Oxford University Press, Oxford, pp. $296-309$. 53. Vid, Hoogenboom, Bob (2006) «Grey Intelligence» en Crime, Law and Social Change, vol 45, pp. 373-381. 
sas que prestan servicios relacionados con la inteligencia de forma casi artesanal y a medida del cliente. De hecho, incluso existen varias asociaciones profesionales de estas empresas que desarrollan importantes labores de lobby en Washington. Este complejo suministra todos los tipos de inteligencia tradicionales del tipo HUMINT, SIGMINT o IMINT ${ }^{54}$ e incluso funciones encubiertas (recordemos que en la guerra de Iraq llegaron a subcontratarse incluso servicios privados de interrogatorio a prisioneros de guerra ${ }^{55}$ ). Las razones aducidas para la externalización de estos servicios, son el ahorro en los costes y una supuesta mayor eficiencia en la prestación de los servicios encomendados. Pero dados que los fondos destinados a inteligencia no han dejado de incrementarse en los últimos 15 años y muy en especial los asignados a este tipo de empresas todo parece indicar que son causas de índole política las que priman a la hora de decidir la contratación externa. La externalización parece ser usada como una forma de eludir las responsabilidades políticas que se pudieran derivar de un mal uso de los servicios de inteligencia, al tiempo que se evita la necesidad de informar a parlamentos y órganos de control de los fines para los que son usados. No hay que excluir tampoco la presión que ejercen este tipo de empresas a través de los lobbies parlamentarios para conseguir contratos del gobierno, muchos de ellos camuflados dentro de contratos genéricos de servicios de defensa ${ }^{56}$. No se debe obviar, como antes apuntamos, que muchas de estas empresas de inteligencia son a su vez filiales de las grandes contratistas del Pentágono ${ }^{57}$.

La otra gran forma de privatización usada para los servicios de inteligencia, y que en nuestra opinión constituye la verdadera privatización de la inteligencia es la retirada del estado de la prestación de dichos servicios. Al igual que buena parte de las guerras modernas, como las que se libran en África por el control de recursos mineros, son libradas por compañías privadas ${ }^{58}$, sin intervención o con intervención residual por parte de los estados, la inteligencia para llevar a cabo estas guerras se lleva a cabo con medios propios o también subcontratados por parte de estas

54. Siglas en inglés para «Human Intelligence» e «Imaginery Intelligence» respectivamente

55. Una crítica al abuso de las empresas privadas de inteligencia para fines moralmente discutibles y una defensa de su estricta regulación puede verse en: Chesterman, Simon (2008) “'We Cant't Spy....If We Can't Buy': The Privatization of Intelligence and the Limits of Outsourcing 'Inherently Governmental Function'» en European Journal of International Law, vol. 19, n5, pp. 1055-1074.

56. Vid.,Hansen, Morten (2014) «Intelligence Contracting: On the Motivations, Interests, and Capabilities of Core Personnel Contractors in the US Intelligence Community» en Intelligence and National Security, vol. 29, nํㅜ 1, pp. 58-81

57. Vid, Melman, Selman (1972) El capitalismo del Pentágono, Siglo XXI, México.

58. Vid, Singer,Peter (2003) Corporate Warriors: The Rise of Privatized Military Industry, Cornell University Press, Ithaca; Mandel,Robert (2002) Armies without States. The Privatization of Security, Lynne Riener Publishers, Boulder. 
modernas empresas bélicas ${ }^{59}$. Pero en el ámbito donde es más perceptible la retirada del estado es en la llamada inteligencia económica, la referida a las necesidades de análisis y tratamiento de información para las empresas y organizaciones no gubernamentales ${ }^{60}$. La inteligencia o contrainteligencia referida a las necesidades de las empresas y organismos no estatales está siendo poco a poco desplazada del ámbito público y descansando en cada vez mayor medida en empresas especializadas en el tratamiento de información especializada, que puede ir desde elementos de contraespionaje industrial a análisis de las fortalezas y debilidades ocultas de los competidores, pasando por el análisis de riesgo país, tanto en el aspecto político como en el económico. El carácter multinacional tanto de la organización empresarial y sus directivos como de su accionariado dificulta cada vez más la identificación de estas modernas organizaciones con el interés nacional de un país en exclusiva y orienta en cada vez mayor medida la prestación de estos servicios hacia la inteligencia privada o mixta ${ }^{61}$. La retirada del estado no sólo puede ser inducida por estas razones. Como antes se apuntó los servicios de inteligencia son administraciones como las demás y están tan sometidas como estas a los vaivenes de los ciclos económicos y a la coyuntura económica particular de cada momento y de cada estado en concreto. Esto es determinadas funciones que antes eran exclusivas del estado ahora se van desplazando hacia el sector privado bien simplemente porque las agencias estatales de inteligencia no tienen fondos suficientes pues no son inmunes a los recortes presupuestarios, bien por falta de personal especializado, bien porque las necesidades de inteligencia ya no son la adecuadas para un estado-nación típico ${ }^{62}$. Por último, los cambios $^{63}$, como ahora veremos, en la recolección y tratamiento de información en el ámbito de la inteligencia ha conducido hacia formas privadas de su tratamiento.

\section{La revolución de las fuentes abiertas y la privatización de la inteligencia.}

Los procesos de privatización de la inteligencia se ven reforzados además de por los factores arriba indicados por la cada vez mayor facilidad de obtención de infor-

59. Sobre el caso español vease: Laguna Sanquirico, Francisco (2009) «Delimitación conceptual relativa a la presencia privada en el marco estatal de la defensa» en Helena Torroja Mateu y Sonia Güell Peris (dirs.) La privatización del uso de la fuerza armada, Bosch, Barcelona, pp. 37-61.

60. Vid.,Laqueur, Walter (1985) A World of Secrets: The Uses and Limits of Intelligence, Basic Books, New York,pp. 38-68.

61. Vid., Martín Barbero, Isaac (2007) «Inteligencia económica: tan lejos, tan cerca» en Inteligencia y Seguridad, no 2, junio-noviembre, pp. 107-120.

62. Vid., Bean, Hamilton (2016), «Privatizing Intelligence» en Rita Abrahamsen y Anna Leander (eds), Routledge Handbook of Private Security Studies, Routledge, New York, pp. 79-88.

63. Vid.,Berkowitz Bruce D. y Goodman, Allan E. (2000) Best truth. Intelligence in the Information Age, Yale University Press, New Haven. 
mación a través de fuentes abiertas que son cada vez más respetadas en el mundo de la inteligencia ${ }^{64}$. Si ya desde los comienzos de la moderna inteligencia esta había descansado en buena medida en el análisis de fuentes abiertas, este proceso se ha acelerado en los últimos quince años con el abaratamiento de su obtención gracias a la revolución en las tecnologías de información y comunicación ${ }^{65}$ y muy especialmente a la difusión a nivel mundial de internet ${ }^{66}$ y muy en especial en su versión 2.0 que incluye la difusión de redes sociales de todo tipo. Nunca ha sido tan fácil obtener información de personas y empresas como en la actualidad. Este abaratamiento ha permitido la aparición de un gran número de empresas que con una simple conexión pueden acceder a enormes cantidades de información que debidamente tratada y analizada ofrece a su vez una inteligencia de gran calidad ${ }^{67}$. Y no sólo permite inteligencia documental, sino que seguimiento de señales, de imágenes o incluso de personas pueden realizarse con precisión desde la pantalla de un ordenador. Este tipo de procesos ha motivado, como señala Díaz Fernández (2013) un gran crecimiento de las agencias privadas que trabaja y procesan información en el sector de la inteligencia. De hecho, programas como Google Earth o Google Maps, por usar ejemplos de programas a los que cualquier internauta puede acceder con facilidad, usados profesionalmente pueden incluso detectar movimientos de armamento o la existencia de bases militares antes ocultas. Las redes sociales tipo Facebook ${ }^{68}$,permiten al minero de datos obtener todo tipo de información sobre un individuo sin mucho esfuerzo y si bien no eliminan del todo la pertinencia del Humint sí que ahorran costes y hacen menos necesarias buena parte de sus funciones. La digitalización de archivos y documentos, incluso confidenciales como se pudo comprobar en Wikileaks, combinados con motores de búsqueda refinados permiten elaborar informes estratégicos de casi cualquier tema en muy poco tiempo. Pero al mismo tiempo nunca las empresas y

64. Vid., Mercado,Stephen C. (2004) «Sailing the Sea of OSINT in the Information Age» en Studies in Intelligence, vol. 48, no 3; McGill, G.M. (Mert) (1994) «OSCINT and the Private Information Sector» en International Journal of Intelligence and CounterIntelligence, vol. 7, no 4, pp. 435-443.

65. Vid.,Navarro Bonilla, Diego (2005)«Medios tecnológicos e Inteligencia: Bases para una interrelación convergente» en Arbor, CLXXX, no 709, enero, pp. 289-313, Gimenez Toledo Elea y Román Román, Adelaida (2001)«Vigilancia tecnológica e inteligencia competitiva: Conceptos, profesionales y fuentes de información» en El profesional de la información, vol. 10, no 5, mayo, pp. 11-20.

66. Vid.,Martín de Santos, Inés y Martín Vega, Arturo (2010) «Las fuentes abiertas de información. Un sistema de competencia perfecta» en Inteligencia y Seguridad, no 8, junio-noviembre, pp. 91-112.

67. No en vano se están acuñando a gran velocidad nuevos términos como el de Guerra 4.0 para incluir los nuevos riesgos de los ataques desde la red como mecanismo de desestabilización. Del mismo modo que se instituyen redes y cuerpos de defensa frente a este tipo de amenazas como es el caso del CNPIC (Centro Nacional de Protección de Infraestructuras Críticas) en España. Este tipo de desarrollos teóricos e institucionales no deja de ser la muestra del cambio en el paradigma de defensa forzado por los cambios tecnológicos.

68. Vease la aplicación de redes sociales de este tipo a las necesidades de la inteligencia económica en: Carvalho, Andrea (2007)«Comunidades virtuales y producción de Inteligencia Económica y Competitiva» en Inteligencia y Seguridad, no 3, 2007, pp. 13-42. 
estados fueron tan vulnerables a la difusión de información de carácter estratégico y nunca fue por tanto la necesidad de servicios de contrainteligencia como hoy ${ }^{69}$, muy en especial en el ámbito de la inteligencia económica ${ }^{70}$ y tecnológica ${ }^{71}$. Dada esta necesidad y la falta de adecuación de los servicios de inteligencia estatales a estas funciones, el estado ha ido poco a poco retirándose de este sector de rapidísima evolución y permitiendo al sector privado que se encargue de la prestación de estas funciones. El estado en este aspecto juega también aquí un papel cada vez más residual, dedicándose a la elaboración de inteligencia en las áreas más vulnerables de la seguridad nacional sobre todo en la protección frente a ataques informáticos potencialmente hostiles y a la protección de secretos en el ámbito militar y, en la medida en que la seguridad nacional se centra en la defensa frente al crimen organizado, en la inteligencia policial. No sólo esto, si bien no se ha abordado en texto estos nuevos actores en el escenario internacional también cuentan con aparatos de inteligencia propios $^{72}$. Guerrillas, piratas o mafias cuentan con sofisticados sistemas de recogida y tratamiento de la información como así lo hacen iglesias o empresas. La revolución tecnológica de las fuentes abiertas es también una revolución en la forma de elaborar inteligencia e incluso en el contenido y en la titularidad de la misma, lo que al abaratarla facilita a estos actores el acceso a la misma y por tanto la extensión de la comunidad de inteligencia a cada vez más organizaciones al tiempo que amplía el número de organizaciones a vigilar.

\section{Conclusión}

El estado moderno se configuró en su momento en relación a la guerra y la guerra hizo al estado moderno ${ }^{73}$ y por tanto los servicios de inteligencia se adaptaron paulatinamente a las necesidades de la misma. Si en su momento la aparición de nuevas tecnologías bélicas permitió a los estados-nación configurarse como los monopolistas

69. Vid., Esteban Martínez, Mario (2010) «¿Por qué debo usar la inteligencia competitiva en mi empresa?» en Inteligencia y Seguridad, no 7, Diciembre -Mayo, pp. 43-55.

70. Vid.,Cruz Beltrán, José Luis (2012)«El perfil del nuevo analista de inteligencia del siglo XXI: De la inteligencia <<tradicional>> hacia la inteligencia económica; y outsourcing o externalización de los servicios de inteligencia» en Fernando Velasco y Rubén Arcos (eds.) Cultura de Inteligencia: Un elemento para la reflexión y colaboración internacional, Plaza y Valdés, Madrid, pp. 245-273; Juillet,Alain (2006) «Principios y aplicación de la Inteligencia Económica» en Inteligencia y Seguridad, no 1, diciembre, pp. 113-135; Silveira de Oliveira, Marcos Aurelio (2007) «Inteligência competitiva: A proteção do conhecemento» en Revista da Escola Superior de Guerra, vol. 23, no 47, janeiro-julho, pp. 85-102.

71. Vid., Blanco Serrano,José Antonio (2009) «Fuentes abiertas de información y elaboración de inteligencia tecnológica para la toma de decisiones» en Diego Navarro y Fernando Velasco (eds.) El alma de la victoria. Estudios sobre Inteligencia Estratégica, Plaza y Valdés, Madrid, pp. 255-265.

72. Vid., Gill, Peter y Pythyan, Mark (2018), Intelligence in an Insecure World, 3rd Ed, Polity, Cambridge.

73. Vid., Porter, Bruce D. (1994) War and the Rise of the State, The Free Press, New York. 
de la guerra y la seguridad frente a sus rivales privados, los cambios en la tecnología y la economía están erosionando el papel central de los estados en lo que se refieren a la producción de seguridad y esta erosión se refiere también a la producción de inteligencia como componente necesario de la misma. Los servicios de inteligencia no pueden ser inmunes a los cambios en las instituciones que las rodean ni tampoco son inmunes a sus coyunturas específicas ${ }^{74}$. Cambios estructurales en la forma de abordar el crimen o la guerra y coyunturas temporales concretas como las crisis económicas, que afectan como no puede ser de otra manera a las administraciones públicas, están obligando a volver a adoptar y adaptar viejas prácticas que parecían olvidadas o residuales, pero con un potencial desconocido en otros tiempos ${ }^{75}$. Las agencias de inteligencia públicas se perciben cada vez más como una mera digresión histórica en la milenaria tradición de espías e informantes y todo apunta a la vuelta, eso sí con tecnologías del siglo XXI y forma corporativa, de la tradicional y artesanal forma de producir inteligencia como industria o profesión liberal especializada. Estos cambios no deberían coger por sorpresa a las actuales agencias de inteligencia que deberían desde este momento comenzar a diseñar y facilitar la transición hacia estos nuevas pero antiguas realidades profesionales.

\section{Bibliografía}

ABRAHAMSEN, R. y M. C. Williams (2009) «Security Beyond the State: Global Security Assemblages in International Politics» en International Political Sociology, vol. 3, pp. 1-17. Doi: 10.1017/cbo9780511974441.008

ADAMS, T. (1999) «The New Mercenaries and the Privatization of Conflict» en Parameters, Summer, pp. 103-116;

ALEJANDRE, L. (2009) «Implicaciones de la presencia privada en el planeamiento y ejecución de la misión militar» en H. TORROJA (dir.) La privatización del uso de la fuerza armada, Bosch Editor, Barcelona, pp. 29-36.

ALEM, J. (1983), El espionaje y el contraespionaje, Fondo de Cultura Económica, México.

ANDERSON, T. y P. J. Hill. (1979) «The Not So Wild Wild West» en Journal of Libertarian Studies, vol. 3, no 1, pp. 9- 29.

AVANT, D. D. (2006) «The Privatization of Security: Lessons from Iraq» en Orbis, vol. 50, no 2, Spring, pp. 1-15. doi: 10.1016/j.orbis.2006.01.009

74. Sin emebargo la visión dominante en mchso académicos sigue siendo la de la seguridad organizada por el estado y sobre una base estatal. Vid., Loch Johnson (2017), National Security Intelligence, Polity, Cambridge.

75. Vid., Lahneman, William J. (2014), «Outsourcing the IC's Stovepipes» en International Journal of Intelligence and Counterintelligence, vol. $16 \mathrm{n} \div 4$, pp. 573-593. 
BAKER, Denae-Peter (2010) Just Warrior Inc.: The Ethics of Privatized Force, Continuum, London.

BEAN, H. (2016), «Privatizing Intelligence» en R. ABRAHAMSEN y A. LEANDER (eds), Routledge Handbook of Private Security Studies, Routledge, New York, pp. 79-88. Doi: 10.4324/9781315850986-9

BENSON, B. (2000) Justicia sin estado. Union Editorial, Madrid.

BERESFORD, M.W. (1957) «The Common Informer, The Penal Statutes and Economic Regulation» en Economic History Review, vol. 10, no 2, pp. 221-238. Doi: $10.2307 / 2590859$

BERKOWITZ, B. D. Y A. GOODMAN (2000) Best truth. Intelligence in the Information Age, Yale University Press, New Haven.

BLANCO SERRANO, J. A. (2009) «Fuentes abiertas de información y elaboración de inteligencia tecnológica para la toma de decisiones» en D. NAVARRO Y F. VELASCO (eds.) El alma de la victoria. Estudios sobre Inteligencia Estratégica, Plaza y Valdés, Madrid, pp. 255-265. Doi: 10.5211/9788492751136.ch10

BRYDEN, A. (2006) "Approaching the Privatisation of Security from a Security Governance Perspective» en A. BRYDEN y M. CAPARINI (eds.), Private Actors and Security Governance, Transaction, New Brunswick, pp. 3-19.

BURMESTER, H.C. (1978) «The Recruitment and Use of Mercenaries in Armed Conflicts» en American Journal of International Law, vol. 72, no 1, January, pp. 37-56. Doi: 10.2307/2199702

CARVALHO, A. (2007) «Comunidades virtuales y producción de Inteligencia Económica y Competitiva» en Inteligencia y Seguridad, no 3, 2007, pp. 13-42. Doi: 10.5211/iys.3.article3

CHESTERMAN, S. (2008) «'We Cant't Spy....If We Can't Buy”: The Privatization of Intelligence and the Limits of Outsourcing 'Inherently Governmental Function'» en European Journal of International Law, vol. 19, n5, pp. 1055-1074.

CLARKE, R. A. y R. KNAKE (2011), Guerra en la red: Los nuevos campos de batalla, Ariel, Barcelona.

CODEVILLA, A. (1992) Informing Statecraft, The Free Press, New York.

CONTAMINE, P. (1984) La guerra en la Edad Media, Labor, Madrid, 1984.

CRUZ-BELTRÁN, J. L. (2012) «El perfil del nuevo analista de inteligencia del siglo XXI: De la inteligencia <<tradicional >> hacia la inteligencia económica; y outsourcing o externalización de los servicios de inteligencia» en F. VELASCO y R. ARCOS (eds.) Cultura de Inteligencia: Un elemento para la reflexión y colaboración internacional, Plaza y Valdés, Madrid, pp. 245-273

DÍAZ FERNÁNDEZ, A. (2013) El papel de la inteligencia estratégica en el mundo actual. En Cuadernos de Estrategia, 162, 35-66. 
DONAHUE, J. D. (1991) La decisión de privatizar. Fines públicos y medios privados, Paidós, Barcelona.

DORFFI, C. (1979) «San Francisco’s Hired Guns» en Reason, August, pp. 26-33.

DOYLE, A. (2010), La compañía blanca, Valdemar, Madrid.

ESTEBAN MARTÍNEZ, M. (2010) «¿Por qué debo usar la inteligencia competitiva en mi empresa?» en Inteligencia y Seguridad, no 7, diciembre -mayo, pp. 43-55.

FEELEY, M. M. (1991) «The Privatization of Punishment in Historical Perspective» en W. T. GORMLEY (ed.) Privatization and Its Alternatives, University of Wisconsin Press, Maison, 1991, pp. 199-225.

GARCÍA GUINDO, M. (2013) «El concepto de Insurgencia a debate, una aproximación teórica». En Revista de Investigaciones Políticas y Sociológicas. número 12 (1), pp. 211-224.

GARCÍA GUINDO, M. (2014). Movimientos insurgentes: el papel, capacidades y respuestas de los Estados». En Revista Política y Estrategia, número 123, pp. 35-50. Doi: 10.26797/rpye.v0i123.57

GILL, P. Y M. PHYTHYAN (2018), Intelligence in an Insecure World, 3rd Ed., Polity, Cambridge.

GIMENEZ-TOLEDO, E. Y A. ROMÁN, (2001) «Vigilancia tecnológica e inteligencia competitiva: Conceptos, profesionales y fuentes de información» en El profesional de la información, vol. 10, no 5, mayo, pp. 11-20.

GOLDHAMMER, H. (1987) The Advisor, Elsevier, New York.

HANSEN, M. (2014) «Intelligence Contracting: On the Motivations, Interests, and Capabilities of Core Personnel Contractors in the US Intelligence Community» en Intelligence and National Security, vol. 29, $\mathrm{n}^{\circ}$ 1, pp. 58-81. Doi: $10.1080 / 02684527.2012 .703044$

HERMAN, Michael (2005) «Intelligence and the Revolution in Military Affairs» en M. HERMAN, Intelligence Services in the Information Age, Frank Cass, London, pp. 49-64.

HORAN, J. (1967) The Pinkertons: The Detective Dynasty That Made History, Bonanza Books, New York. Doi: 10.1353/cwh.1970.0001

HOLCOMBE, R. (2004) «Government: Unnecessary but Inevitable» en Independent Review, vol. 8, no 3, Winter, pp. 325-342 doi: 10.4324/9781315082349-22

HOOGENBOOM, B. (2006) «Grey Intelligence» en Crime, Law and Social Change, vol 45, pp. 373-381.

JOHNSON, L. (2017), National Security Intelligence, Polity, Cambridge.

JOHNSTON, L. (1992) The Rebirth of Private Policing, Routledge, London.

JUILLET, A. (2006) «Principios y aplicación de la Inteligencia Económica» en Inteligencia y Seguridad, $\mathrm{n}^{\circ}$ 1, diciembre, pp. 113-135. Doi: 10.5211/iys.1.article8 
KEMPA, M. (2016), "The Private Origin of Modern Public Policing» en R. ABRAHAMSEN y A. LEANDER (eds.), Routledge Handbook of Private Security Studies, Routledge, New York, pp. 20-29. Doi: 10.4324/9781315850986-3

KEEFE, P. (2010), «Privatized Spying: The Emerging Intelligence Industry» en L. JOHNSON (edit.), The Oxford Handbook of National Security Intelligence, Oxford University Press, Oxford, pp. 296-309. Doi: 10.1093/ oxfordhb/9780195375886.003.0018

LAGUNA-SANQUIRICO, F. (2009) «Delimitación conceptual relativa a la presencia privada en el marco estatal de la defensa» en H. TORROJA y S. GÜELL (dirs.) La privatización del uso de la fuerza armada, Bosch, Barcelona, pp. 37-61.

LAHNEMAN, W. (2010) «The Need for a New Intelligence Paradigm» en International Jounal of Intelligence and Counter Intelligence, vol. 23, pp. 201-225.

LAHNEMAN, W. J. (2014), «Outsourcing the IC's Stovepipes» en International Journal of Intelligence and Counterintelligence, vol. $16 \mathrm{n}^{\circ}$ 4, pp. 573-593. Doi: 10.1080/716100468

LAQUEUR, W. (1985) A World of Secrets: The Uses and Limits of Intelligence, Basic Books, New York,pp. 38-68.

LIAROPOULOS, A. (2006) A (R)evolution in Intelligence Affairs? In Search of a New Paradigm, Rearch Paper $n^{\circ} 100$, Research Institute for European and American Studies, Athens, June.

LIND, W., J. SCHMITT, W. SUTTON Y G. WILSON. (1989) «The Changing Face of War» en Marine Corps Gazette, October, pp. 22-26.

LITTLE, C. B. Y C. SHEFFIELD (1983) «Frontiers and Criminal Justice: English Private Prosecution Societies and American Vigilantism in the Eighteen and Nineteen Centuries» en American Sociological Review, vol. 48, n 6, December, pp. 796-808. Doi: 10.2307/2095326

LYNCH, T. y A. J. WALSH, (2000) «The Good Mercenary» en Journal of Political Philosophy, vol. 8, no 2, pp. 133-153.

MABEE, B. (2009), «Pirates, Privateers and the Political Economy of the Violence» en Global Change, Peace \& Security, vol. 21, no 2, June, pp. 139-152. Doi: $10.1080 / 14781150902871994$

MANDEL, R. (2002) Armies without States. The Privatization of Security, Lynne Riener Publishers, Boulder.

MARKUSEN, A. R. (2003) «The Case Against Privatizing National Security» en Governance, vol. 16, no 4, October, pp. 471-501. Doi: 10.1111/1468-0491.00225

MARTÍN-BARBERO, I. (2007) «Inteligencia económica: tan lejos, tan cerca» en Inteligencia y Seguridad, no 2, junio-noviembre, pp. 107-120. Doi: 10.5211/ iys.2.article7 
MARTÍN DE SANTOS, I. y A. MARTIN, (2010) «Las fuentes abiertas de información. Un sistema de competencia perfecta» en Inteligencia y Seguridad, no 8, junionoviembre, pp. 91-112. Doi: 10.5211/iys.8.article5

MCGILL, G.M. (Mert) (1994) «OSCINT and the Private Information Sector» en International Journal of Intelligence and Counter Intelligence, vol. 7, $\mathrm{n}^{\circ} 4$, pp. 435443. Doi: $10.1080 / 08850609408435262$

MELMAN, S. (1972) El capitalismo del Pentágono, Siglo XXI, México.

MERCADO, S. C. (2004) «Sailing the Sea of OSINT in the Information Age» en Studies in Intelligence, vol. 48, $\mathrm{n}^{\circ}$ 3. Doi:10.1037/e741272011-005

MINOW, M. (2005) «Outsourcing Power: How Privatizing Military Efforts Challenges Acountability, Professionalism and Democracy» en Boston College Law Review, vol. 46, n 5 , pp. 989-1026.

MONSALVE, R. (2012) «¿Hacia una inteligencia Europea?» en J.J. FERÁNDEZ, D.SANSÓ-RUBERT PASCUAL, J. PULIDO Y R.MONSALVE (eds.) Cuestiones de inteligencia en la sociedad contemporánea, Ministerio de Defensa, Madrid, pp. 121-126.

NAVARRO, D. (2005) «Medios tecnológicos e Inteligencia: Bases para una interrelación convergente» en Arbor, CLXXX, nº 709, enero, pp. 289-313. Doi: 10.3989/arbor.2005.i709.508

NAVARRO, D. (2009). ¡Espías!: tres mil años de información y secreto, Plaza y Valdés, Madrid.

NOZICK, R. (1988) Anarquía, estado y utopía, Fondo de Cultura Económica, México. NUÑEZ-VILLAVERDE, J. A. (2009) «Privatización e internacionalización del uso de la fuerza: los mercenarios como lacra del marco de seguridad del siglo XXI» en H. TORROJA (dir.) La privatización del uso de la fuerza armada, Bosch Editor, Barcelona, pp. 63-82.

O’TOOLE, G. (1978) The Private Sector: Private Spies, Rent -a-Cops, and the Police-Industrial Complex, W.W. Norton, New York, pp. 20-40. https://doi. org/10.1016/0007-6813(78)90079-4

OHMAE, K. (1995) The End of the National State; The Rise of Regional Economics, The Free Press, New York.

OLIER ARENAS, E. (2013) "Inteligencia estratégica y seguridad económica» en Cuadernos de Estrategia, no 162, pp. 9-33.

PORTER, B. D. (1994) War and the Rise of the State, The Free Press, New York.

POZO SERRANO, P. (2008), «La privatización de la formación militar y policial en los programas internacionales de reforma del sector de la seguridad» en FERNÁNDEZ RODRÍGUEZ, J. J., JORDÁN ENAMORADO, J. y SANSÓRUBERT PASCUAL, D. (eds.) Seguridad y defensa hoy: Construyendo el futuro. Plaza y Valdés, Madrid. 
PULIDO GRAGERA, J. (2010) «Inteligencia: la savia de la Unión Europea» en Inteligencia y Seguridad, no 7, Diciembre-Mayo, pp. 87-107. https://doi. org/10.5211/iys.7.article5

RATHMELL, A. (1998). «Privatising Intelligence» en Cambridge Review of International Affairs, vol. XI, no 2, Spring, pp. 199-211. https://doi. org/10.1080/09557579808400199

REISS Jr., A. J. (1991) «Private Employment of Public Police» en William T. Gormley (ed.) Privatization and Its Alternatives, University of Wisconsin Press, Maison, pp. 226-240.

RICKARDS, J. (2012). Currency Wars. Penguin, New York. https://doi. org/10.1177/089124167300200310

RUIZ MIGUEL, C. (2002). Servicios de inteligencia y y seguridad del estado constitucional, Tecnos, Madrid, 2002.

SAVAS, E.S. (2001). Privatization and Public Private Partnerships, East-West Press, New Delhi,

SCAHILL, J. (2010). Blackwater: El auge del ejército mercenario más poderoso del mundo, Planeta, Barcelona.

SCHEREIER, F. y CAPARINI, M. (2005). Privatising Security: Law, Practice and Governance of Private Military and Security Companies. Geneva Center for the Democratic Control of Armed Forces, Geneva.

SETTON, K. M. (1985). Los catalanes en Grecia. Orbis, Barcelona.

SHAPIRO, S. S. (1987) «Policing Trust» en SHEARING, C. D. y STENNING, P. C. (eds.) Private Policing. Sage, London, 1987, pp. 194-220.

SHEARER, D. (1988). «Outsourcing War» en Foreign Policy, n 112, Fall, pp. 68-81. https://doi.org/10.2307/1149036

SHORROCK, T. (2009). Spies for Hire: The Secret World of Intelligence Outsourcing. Simon \& Schuster, New York.

SILVEIRA DE OLIVEIRA, M. A. (2007) «Inteligência competitiva: A proteção do conhecemento» en Revista da Escola Superior de Guerra, vol. 23, no 47, janeirojulho, pp. 85-102.

SINGER, P. (2003). Corporate Warriors: The Rise of Privatized Military Industry, Cornell University Press, Ithaca. https://doi.org/10.7591/9780801459894

SMITH, E. B. (2003) «The New Condottieri and US Policy: The Privatization of Conflict and Its Implications» en Parameters, Winter, pp. 104-119. https://doi. org/10.21236/ada402135

SOUTH, N. (1987). «Law, Profit, and Private Persons» en SHEARING, C. D. y P. C. STENNING (eds.) Private Policing, Sage, London, pp. 72-109.

STROMBERG, J. (2003). "Mercenaries, Guerrillas, Militias, and the Defense of Minimal States and Free Societies» en HOPPE. H. H. (ed.). The Myth of National Defense. Ludwig von Mises Institute, Auburn, pp. 215-238. 
TABARROK, A. (2007). «The Rise, Fall and Rise Again of Privateers» en Independent Review, vol. XI, nº 4, Spring, pp. 565-577.

TRENTO, J. T. (2005). Prelude to Terror: The Rogue CIA and the Legacy of America's Private Intelligence Network. Carroll and Graf Publishers, New York.

TRUJILLO ÁLVAREZ, P. (2009) «La falta de institucionalización de la inteligencia y su impacto en la seguridad: el caso de Guatemala» en Inteligencia y Seguridad, $\mathrm{n}^{\circ}$ 5, diciembre-mayo, pp. 123-154.

URBINA, J. J. (2008), «El papel de las compañías militares y de seguridad privadas en los conflictos armados recientes: una aproximación al estatuto jurídico de su personal en el derecho internacional humanitario» en FERNÁNDEZ RODRÍGUEZ, J. J.; J.JORDÁN ENAMORADO y D. SANSÓ-RUBERT PASCUAL (eds.). Seguridad y defensa hoy: Construyendo el futuro. Plaza y Valdés, Madrid. VAN CREVELD, M. (1991). The Transformation of War. The Free Press, New York.

VAN CREVELD, M. (1999). The Rise and Decline of the State. Cambridge University Press, Cambridge,

WULF, H. (2007). «Privatizing and Internationalizing Violence» en The Economics of Peace and Security Journal, vol. 2, n ${ }^{\circ}$ 1, pp. 35-40. https://doi.org/10.15355/2.1.35

ZARATE, J. C. (2014), «The Coming Financial Wars» en Parameters, vol. 43, no 4, Winter.

ZIELINSKI, M. (1995). «Armed and Dangerous: Private Police in the March» en Covert Action Quarterly, nº 54, Fall, pp. 44-50. 